\title{
Atomic and Electronic Structures of Functionalized Nanodiamond Particles
}

Shery L. Y. Chang ${ }^{1}$, Christian Dwyer ${ }^{2}$, Katia March ${ }^{1}$, Michel Mermoux $^{3}$, Nicholas Nunn ${ }^{4}$, Olga Shenderova ${ }^{4}$, Eiji Osawa ${ }^{5}$ and Amanda S. Barnard ${ }^{6}$

${ }^{1 .}$ LeRoy Eyring Center for Solid State Science, Arizona State University, Tempe, AZ 85287, United States.

2. Department of Physics, Arizona State University, Tempe, AZ 85287, United States.

3. Universite Grenoble Alpes, and CNRS, LEPMI, F-38000 Grenoble, France.

4. International Technology Center, 8100 Brownleigh Dr., Raleigh, North Carolina 27617, United States

${ }^{5}$.NanoCarbon Research Institute, Ueda, Japan.

6. Virtual Nanoscience Laboratory, CSIRO, Parkville, Australia.

Nanodiamond particles are promising candidates in a variety of applications such as drug delivery [1], diagnostic imaging [2], photo-electrochemical $\mathrm{CO}_{2}$ reduction [3], seeding agents for diamond film growth by chemical vapor deposition [4], and energy storage [5]. The uniformity and purity of surface functionalization is critical in dictating success or failure in these applications. However, due to the complexity of the surfaces, there is a lack of fundamental understanding of the nanodiamond surface structures. Moreover, the surface structures can change during functionalization, since the process can involve, for example, thermal oxidation or hydrogenation at high temperatures.

Here we address this challenge by using the advanced imaging and spectroscopy in the TEM. We examined diamond particles synthesized using the detonation (termed detonation nanodiamond, DND) and high pressure high temperature (HPHT) processes. The former method produces particles typically less than $5 \mathrm{~nm}$, while the latter produces particles from tens to hundreds of $\mathrm{nm}$. A monochromated, aberration-corrected TEM (Titan, FEI Company) operated at $80 \mathrm{kV}$ was used for quantitative determination of the atomic structures. A monochromated, aberration corrected dedicated STEM (Nion UltraSTEM $^{\mathrm{TM}} 100$ ) operated at $60 \mathrm{kV}$ was used for electron energy loss spectroscopy to determine the electronic structures. The low electron beam energies minimize atomic structural damage.

The benefits of monochromation and aberration-correction for imaging at $80 \mathrm{kV}$ can be clearly seen in Fig. 1, where near $1 \AA$ spatial resolution provides unambiguous experimental evidence of the surface structure. The as-synthesized detonation nanodiamond particles show fullerene-like surface structures, which is the result of surface relaxation and the small particle size (Fig. $1(a-b))$. This surface behavior is also predicted by our density functional tight-binding calculations (for nanodiamond particle with same size range observed in our experiment). Upon hydrogenation treatment, the DND surface was found to be graphitized, as evidenced by the two surface graphitic-like layers (Fig. 1(c)).

Fig. 2 shows experimental carbon K-edge spectra from DND, along with calculated spectra from density functional theory [6]. We have identified the pre-edge peaks $(282.5,284.6$ and $286.5 \mathrm{eV})$ that are associated with vacancies and fullerene-like curved surfaces, respectively. These findings will be compared with both the hydrogenated nanodiamonds and the HPHT nanodiamonds, where the surface structures are distinctly different. Hence this combination of HRTEM and EELS provides a viable route for revealing how surface functionalization alters the atomic and electronic structures of nanodiamonds. 


\section{References:}

[1] E Chow et al, Sci. Trans. Med. 3 (2011) p. 7321.

[2] L Manus et al, Nano Lett. 10 (2010) p. 484.

[3] Liu et al, J. Am. Chem. Soc. 137 (2015), p. 11631.

[4] O A Williams et al, ACS Nano 4 (2010), p. 4824.

[5] W Gu et al, Carbon 53 (2013), p. 292.

[6] S L Y Chang et al, Nanoscale 8 (2016), p. 10548.

(a)

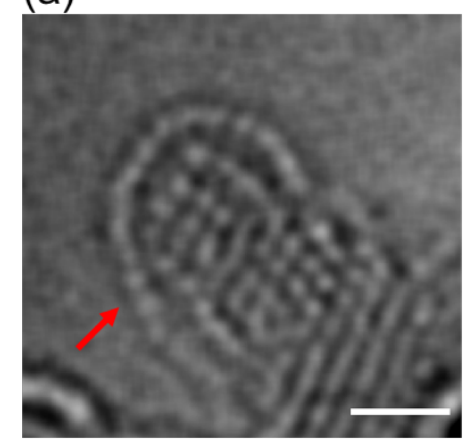

(b)

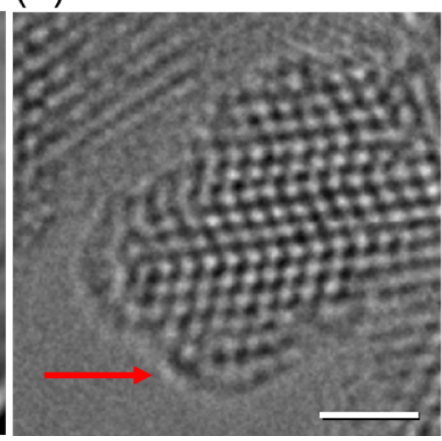

(c)

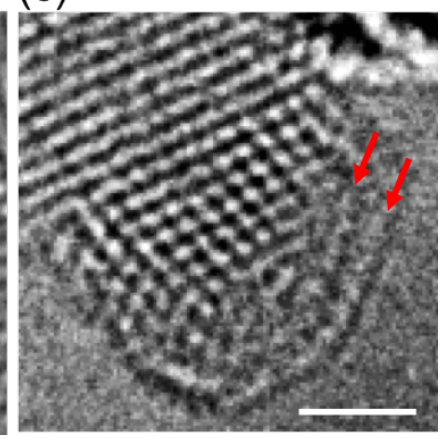

Figure 1. HRTEM images of detonation nanodiamond particles taken using a monochromated, aberration-corrected Titan operating at $80 \mathrm{kV}$. (a-b) As-synthesized nanodiamond, and (c) hydrogenated nanodiamond. Red arrows in (c) indicate the surface graphitic-like layer(s).

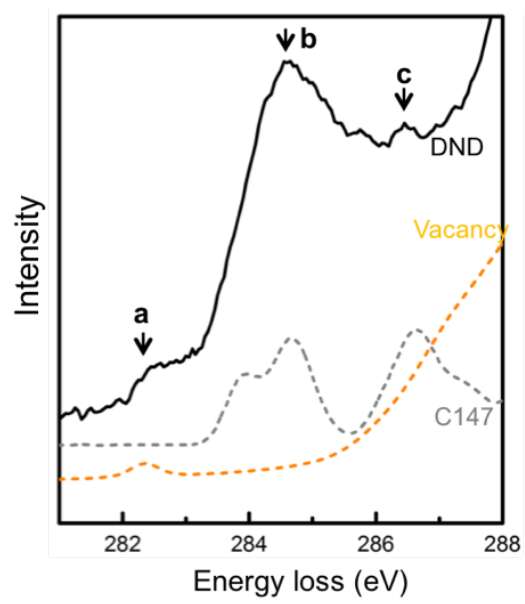

Figure 2. Monochromated carbon K-edge EEL spectra from DND particles, showing pre-edge peaks indicative of vacancies and surface relaxation. Also shown are calculated EEL spectra from a vacancy in the diamond lattice (orange) and a $\mathrm{C}_{147}$ carbon cluster (grey). 